\title{
Confined Space Enables Spontaneous Liquid Separation by Molecular Size: Selective Absorption of Alkanes into a Polyolefin Cast Film
}

Ayano Chiba, ${ }^{* \dagger}$ Akio Oshima, ${ }^{\star}$ and Ryo Akiyama ${ }^{*}$

${ }^{\dagger}$ Department of Physics, Keio University, Hiyoshi 3-14-1, Kohoku-ku, Yokohama 223-8522, Japan
${ }^{\ddagger}$ Department of Chemistry, Kyushu University, 744 Motooka, Nishi-ku, Fukuoka 819-0395, Japan
E-mail: ayano@phys.keio.ac.jp, rakiyama@chem.kyushu-univ.jp

Number of pages: 10

Number of figures: 6

\section{Methods}

\section{Raw data and analysis of x-ray diffraction measurements}

To obtain the blue thin line of Figure 1, we used the raw data shown in Figure S1. The blue thin line in Figure 1 shows the result of simply subtracting the diffraction of hexane alone from the diffraction of the P4MP1 film containing hexane. All the displays in Figure 1 and the subtraction between the data were done without scaling the data against each other.

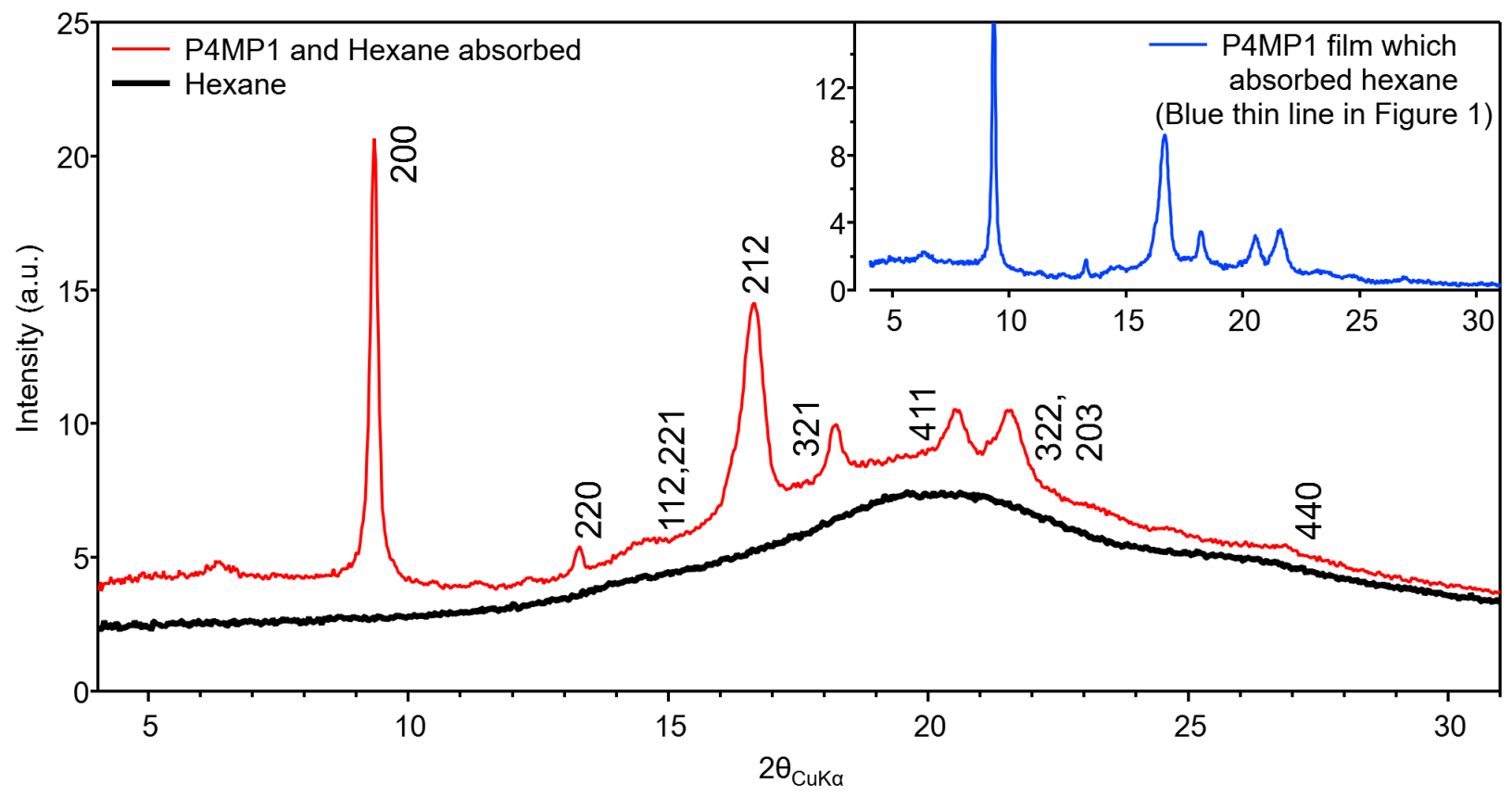

Figure S1. Raw data of x-ray diffraction. The raw data of diffraction patterns of hexane only (denoted by the black thick line) and the film which absorbed hexane (red thin line), both of which contain signals from the Kapton film and the sample folder. The blue line in the inset (which is identical to the blue thin line in Figure 1) shows the result of simply subtracting the raw data shown by the black thick line (hexane) from the raw data shown by red thin line (P4MP1 film with absorbed hexane). 


\section{Raw data and analysis of infrared absorption spectroscopy measurements}

In this section, we show the analysis method of the data of infrared absorption spectroscopy. In similar studies in the past, ${ }^{1}$ time-resolved measurements such as those shown below were not performed. We adopted this method for more accurate evaluation. It should be noted, though, that the conclusions of our article remain the same even if the conclusion is drawn only from the time-resolved first data as in the conventional method without performing the time-resolved measurement.

To obtain Figure 3, we adopted the values obtained by extrapolating the time dependences of peak height to time zero, as shown in Figure S2. Time zero is the time when the film was pulled from the liquid. Figure S2 shows the time dependence of the peak height of the peak around $2100 \mathrm{~cm}^{-1}$ of the infrared absorption spectra. Insets are the raw data of the time-resolved first point, and the arrows show the peak at $2100 \mathrm{~cm}^{-1}$. The peak at $2100 \mathrm{~cm}^{-1}$ can be assigned as the C-D stretching mode, ${ }^{2,3}$ where D denotes deuterium. Although the double exponential was used for the fitting function, the two relaxation coefficients converged to almost the same value.

Similar to the selective absorption of the decane-pentane system shown in Figure 3a, the results are also obtained with the decane-hexane system (Figure S3). Some examples of the raw data for this plot are shown in Figure S4. The bands at 2100 and $2217 \mathrm{~cm}^{-1}$ observed in Figure S4a, b, and c can be assigned to the C-D stretching modes for deuterated hexane (hexane(D)). ${ }^{2,3}$ The band at $721 \mathrm{~cm}^{-1}$ observed in (a), (b), and (d) can be assigned to the $\mathrm{CH}_{2}$ rocking vibration ${ }^{2}$ for normal decane. The clear appearance of the bands at 2100 and $2217 \mathrm{~cm}^{-1}$ in (c) shows that hexane(deuterated, D) is absorbed in the film. The clear band at $721 \mathrm{~cm}^{-1}$ and the weakness of the bands at 2100 and $2217 \mathrm{~cm}^{-1}$ in (a) and (b) shows that decane is selectively absorbed in the P4MP1 film, and hexane is nearly excluded from the film. We may note that, since the peak at $721 \mathrm{~cm}^{-1}$ is not strong enough, this peak was not used to estimate the molar concentration of each solvent in this article. Instead, the amount of decane absorbed was estimated from the peak intensity at $2100 \mathrm{~cm}^{-1}$ by using decane(D)-hexane solvent, examples of which are shown in Figure S2a and c. 

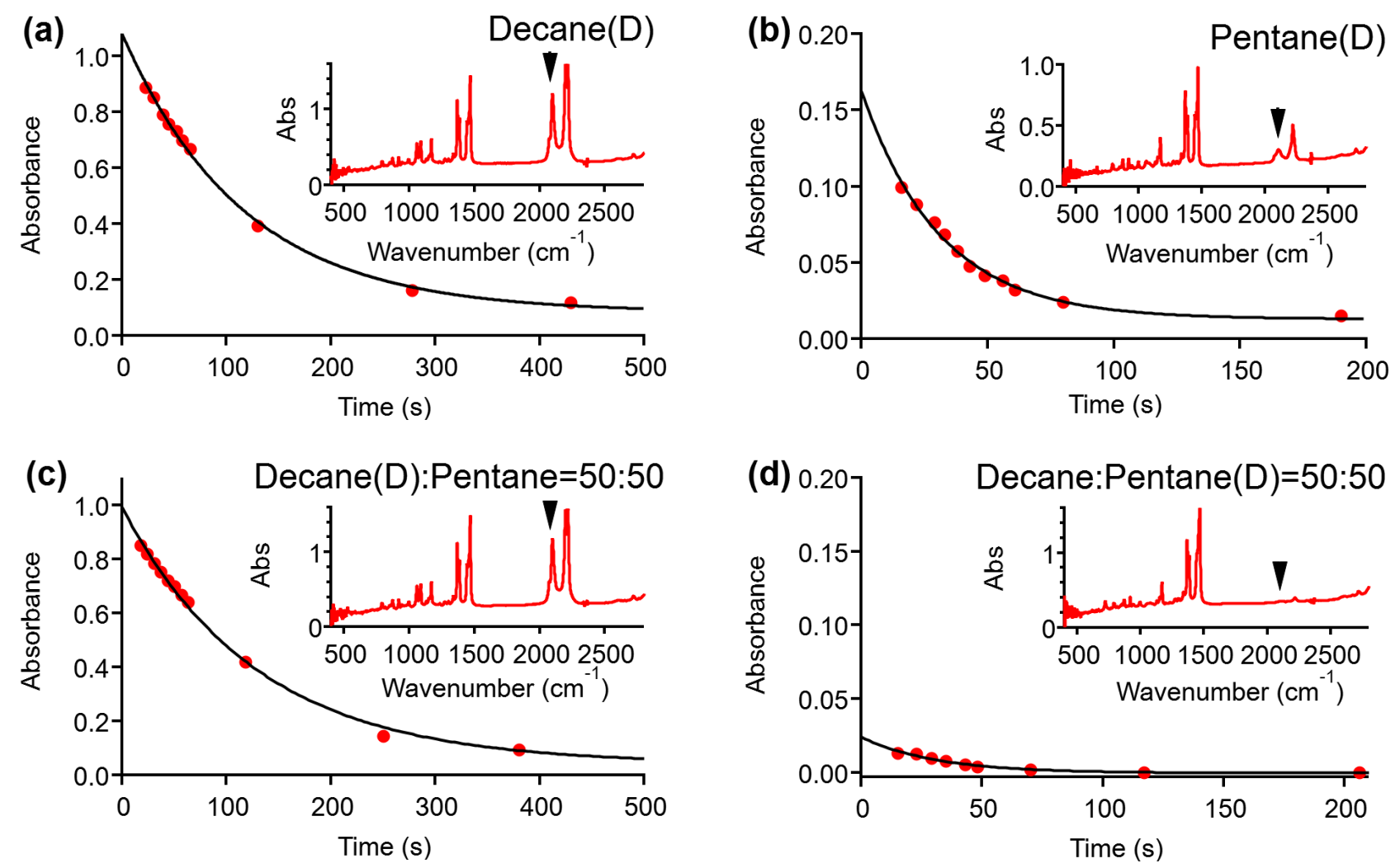

Figure S2. Absorbance changes of P4MP1 film after removal from solvents, with respect to the peak at $2100 \mathrm{~cm}^{-1}$, which is assigned as the C-D stretching mode. The solvents immersed are (a) pure decane(deuterated, D), (b) pure pentane(D), (c) binary mixtures of decane(D) : pentane = 50:50 (volume fraction), and $(\mathrm{d})$ decane : pentane $(\mathrm{D})=50: 50$ (volume fraction). Insets are the raw data of the timeresolved first point, and the arrows show the peak at $2100 \mathrm{~cm}^{-1}$.

Figure S3. Molar concentration of absorbed solvent when P4MP1 as-cast films are immersed in binary mixtures of decane and hexane. All concentrations shown here were estimated from the peak height of the peak at $2100 \mathrm{~cm}^{-1}$, assigned as the C-D stretching mode, in the infrared absorption spectra for the deuterated alkane. The red circles show the result obtained by the measurement of decane(deuterated, D)-hexane binary solvents, and blue squares, that of decane-hexane(D) binary solvents.

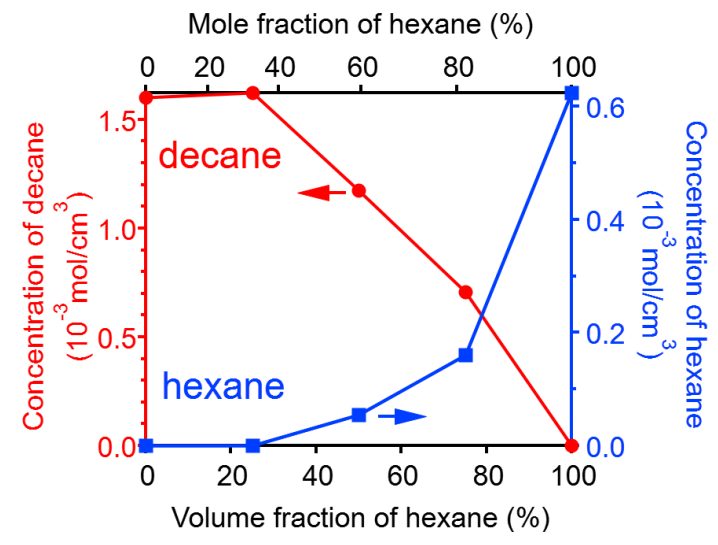




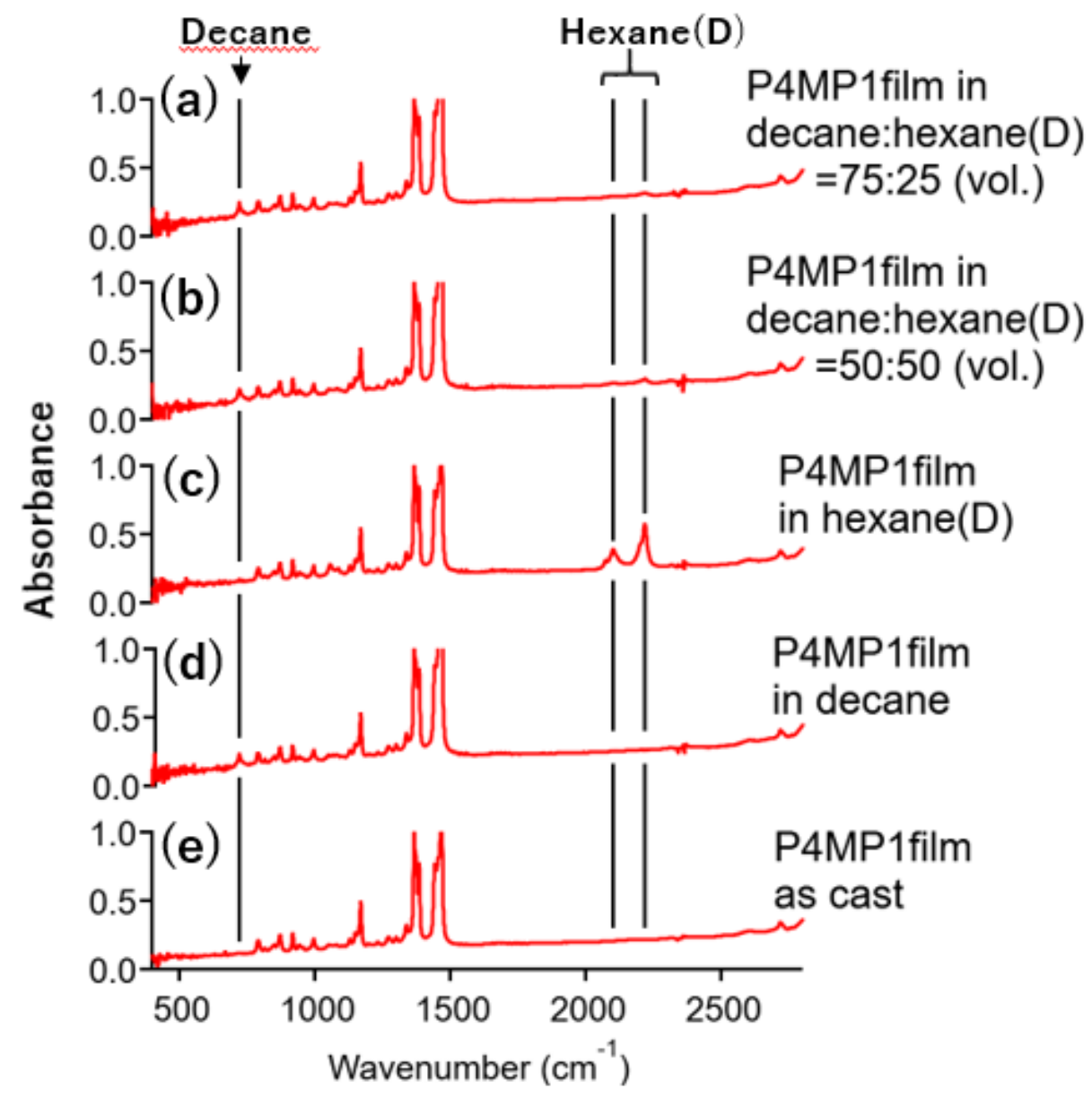

Figure S4. The raw data of the time-resolved first point of infrared absorption spectroscopy measurements of the P4MP1 films immersed in various solvents. The immersed solvents are (a) decane : hexane(D) $=75: 25$ (volume fraction), (b) decane : hexane(D) $=50: 50$ (volume fraction), (c) pure hexane(D), and (d) pure decane. (e) the data for the P4MP1 as-cast film. The bands at 2100 and $2217 \mathrm{~cm}^{-1}$ observed in (a), (b) and (c) can be assigned to the C-D stretching modes for hexane(D). The band at 721 $\mathrm{cm}^{-1}$ observed in (a), (b) and (d) can be assigned to the $\mathrm{CH}_{2}$ rocking vibration for normal decane. 


\section{Supplementary Discussion}

\section{Trial estimation of the local density of guest molecules}

In this section, we would like to show a brief and simple estimation that the longer molecules are selectively absorbed from the point of view of the overlap of excluded volumes.

Let us approximate a methane molecule as a sphere with radius $\sigma$ and consider its association stability with a host tube (Figure 5c). When a tube of inner diameter $\sigma$ is immersed in "liquid methane" with a packing fraction $\phi=0.35$, the overlap of excluded volumes due to methane entering the tube is $\Delta \mathrm{V}=$ $4 \pi \sigma^{3} / 3$. The free energy gain can be estimated from equation (1) as: $\Delta \mathrm{F}=-k_{\mathrm{B}} T \rho \Delta V=-8 k_{\mathrm{B}} T \phi$, where $\rho=\phi / v$ is the number density of methane, and $v=4 \pi(\sigma / 2)^{3} / 3$ is the volume of methane. The local density of methane in the tube is $\mathrm{g}(\boldsymbol{x})=\exp \left(-\Delta \mathrm{F} / k_{\mathrm{B}} T\right)=16$, where $\boldsymbol{x}$ is the position in the host tube.

Assuming that the ethane molecule is twice the length of methane and the overlap of excluded volumes is doubled and the number densities of the guests are the same, the abundance ratio of ethane and methane in the tube becomes $\exp \left(-2 \Delta \mathrm{F} / k_{\mathrm{B}} T\right) / \exp \left(-\Delta \mathrm{F} / k_{\mathrm{B}} T\right)=\exp \left(-\Delta \mathrm{F} / k_{\mathrm{B}} T\right)$. That is, the abundance ratio of longer ethane is expected to be 16 times that of shorter methane in the tube, $16^{2}$ times that of bulk methane. Similar estimates can also be applied to other alkane systems. Since this is an estimate based on the Asakura-Oosawa theory, the actual value deviates from this estimation. However, it is convincing that, when the tube is immersed in the mixed solvent of pentane and decane, a large difference will occur in the probability of finding each molecule in the tube. If decane is strongly preferentially absorbed, pentane will almost be eliminated. 


\section{Supplementary equations S1 and S2: Simple theoretical calculation for Figure 3a}

In this section, we explain the reason why the absorption amount of longer guest molecules is not likely to decrease even if the volume fraction of shorter molecules becomes $50 \%$, e.g., as seen in Figure 3a. In our experiments, we used linear guest molecules of two different lengths, but in this section, we discuss a mixture of hard spheres of two different diameters as guest molecules for simplicity. At the same time, a pore formed by the host molecules is approximated as a tube. In an actual system, the pores are located between the main chains of the polymer and must have more complicated shape, and guest molecules are also not spherical but linear. However, this model is sufficient to explain that large particles are selectively absorbed, and this conclusion will not depend on the details of the guest and host shape.

Before describing the details, note the following. In this section, "volume fraction of small spheres" (which corresponds to the horizontal axis in Figure 3) is replaced with "scaled number density of small spheres". Both are in a proportional relationship in a fixed volume system, i.e., in the system in which the volume after mixing is the sum of the volumes of each liquid before mixing. The binary solvent used in Figure 3 is an example of such a system because there is no increase or decrease in volume due to mixing (Figure 4).

The number density of large particles (e.g., decane in Figure 3a) in the bulk solution monotonously decreases as the number density of small particles (e.g., pentane) increases. Nevertheless, the absorption amount of large particles does not decrease in the experimental result. This can be explained based on the two-component Asakura-Oosawa theory. We will explain it by dividing into two factors: The factors that (i) increase and (ii) decrease the absorption of large molecules when small molecules are added.

(i) The absorption enhancement factor of large particles is as follows. The total particle number density increases as the small particle number density increases, since the total packing fraction is constant as experimentally shown in Figure 4. Here, the total packing fraction means the sum of the packing fractions for both particles. The effective attractive force between the inner wall of the host tube and the larger particles becomes stronger as the total particle number density increases. The reason for the increase of effective attraction is that the stability of large particles in contact with the inner wall increases linearly as the number of small particles increases ${ }^{4}$ (See also Supplementary equation S3). Here, $W(\boldsymbol{x})$ is defined as the effective interaction between the tube and large particles at a position $\boldsymbol{x}$. The stability $\epsilon(>0)$ of the large particle on the inner wall is $-W(\boldsymbol{x})$ at the position in contact with the inner wall. As the stability $\epsilon$ becomes larger, the large particles become more stable on the inner wall. There is a relation between the effective interaction $W(\boldsymbol{x})$ and the reduced density profile $g(\boldsymbol{x})$ of large particles as ${ }^{5}$

$$
g(\boldsymbol{x})=\exp \left\{-\frac{W(x)}{k_{\mathrm{B}} T}\right\} .
$$

To explain the reduced density profile $g(\boldsymbol{x})$ we define the local density $\rho(\boldsymbol{x})$. For example, the number density near the wall becomes larger than the bulk number density $\rho_{\text {Bulk }}$ when the stability near the wall is larger than the bulk. When the number density depends on a position $\boldsymbol{x}, \rho(\boldsymbol{x})$ is defined as the density at the position $\boldsymbol{x}$. The volume integral, therefore, gives the local number at the region. Here, the reduced density profile $g(\boldsymbol{x})$ is defined as a ratio $\rho(\boldsymbol{x})$ to $\rho_{\text {Bulk }}$ as

$$
g(\boldsymbol{x})=\frac{\rho(\boldsymbol{x})}{\rho_{\text {Bulk }}} .
$$

As stated above, if the stability $\epsilon$ linearly increases with the number of small particles, $W(\boldsymbol{x})$ linearly decreases from $\epsilon=-W(\boldsymbol{x})$, and the value of $g(\boldsymbol{x})$ that express the number of large particles in contact with the inner wall surface increases exponentially. This exponential increase is represented by the red dashed line in Figure S5.

Note that the total number of particles increases as the number of small particles increases. The reason is as follows. If one replaces large particles with smaller ones while maintaining total packing fraction, the number of particles increases rapidly. For example, when the size ratio is 2 , the volume of one large particle corresponds to the volume of eight small particles, and if one large particle is reduced, the volume packing ratio will not be constant unless eight small particles are replenished. It is expected that the total packing fraction is almost maintained since decane and pentane have little difference in direct 
interaction and the shape is similar. And experimentally, the total packing fraction is actually constant regardless of the volume fraction for all binary mixtures used in the experiment (Figure 4). In such a case, an increase in the volume fraction of small alkanes results in an increase in the total number of particles.

It is important to note that, when the inner diameter of the tube approaches the large particle diameter, the large particle absorption amount inside the tube can be approximately estimated based on the contact value $g(\boldsymbol{x}=$ contact $)$. Here, $\boldsymbol{x}$ is at the contact position with the inner wall of the tube. In the opposite limit, selective absorption does not occur when the inner diameter of the tube is very large, because the volume not affected by the inner wall dominates.

Next, let us look at the factor (ii), i.e., the reduction factor of large particle absorption as small particles are added. As the number density of small particle increases, the number density of the large particles in the bulk mixture $\rho_{\text {Bulk }}$ decreases linearly, becoming zero when the scaled number density of the small particle is one. This is plotted as a blue dotted line in Figure S5.

The actual adsorption amount is estimated by the product of (i) and (ii) according to equation (S2). That is, the black solid line in Figure S5 gives a rough description of the absorption amount of the large particles experimentally given in Figure 3. However, this plot overemphasizes the effect of contact value. In the case of evaluating the absorption amount, it is necessary to include a portion near the central axis of the tube, far from the wall, which will be close to the abundance ratio of small and large particles in the bulk liquid. Alternatively, it is necessary to include absorption by the bulk ratio to the amorphous part having a larger pore size.

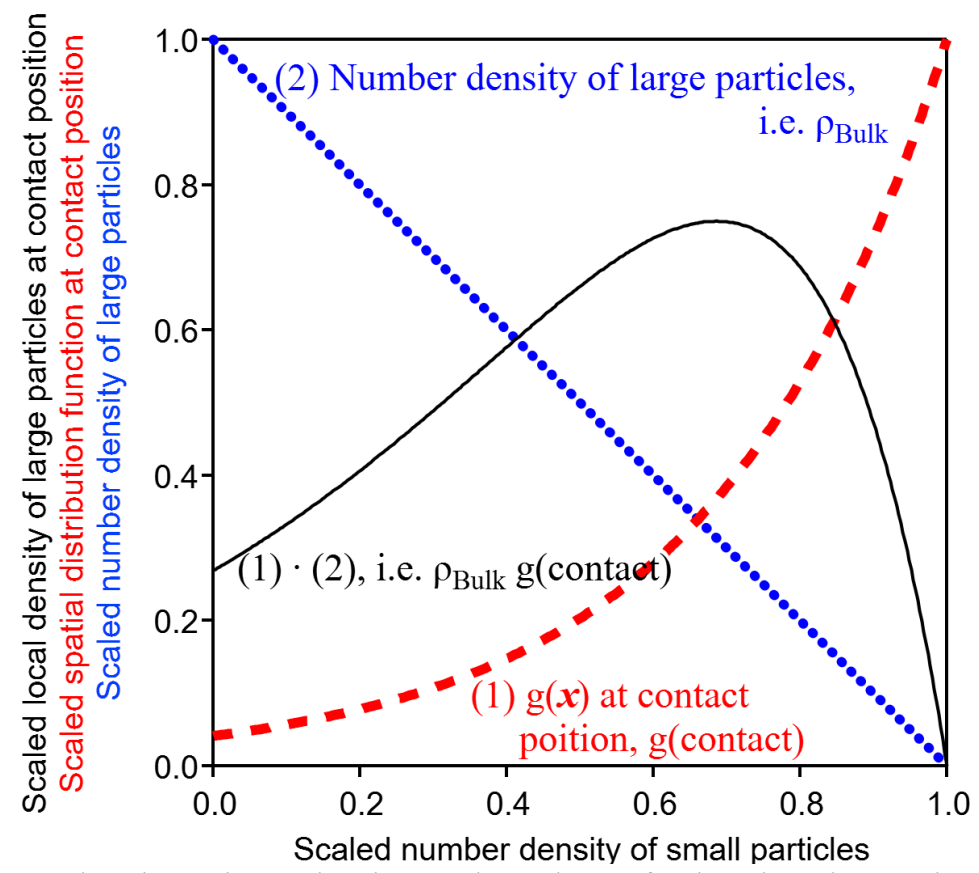

Figure S5. Calculation results that give a basic explanation of why the absorption amount of large molecules does not decrease even if the volume fraction of small molecules increases. Factors that increase (red dashed line) and decrease (blue dotted line) the amount of absorption of large molecules. The probability of finding large particles in a tube immersed in a binary mixed liquid of large and small particles is plotted as a function of the scaled number density of small particles. Scaled local density of large particles at contact position (black solid line), scaled spatial distribution function of large particles at contact position (red dashed line), and scaled number density of large particles of bulk liquid (blue dotted line), as functions of scaled number density of small particles. The increase factor $g(\boldsymbol{x})$ at the contact position is calculated by using the two-component Asakura-Oosawa theory. 
If the inner diameter of the tube is larger ( 5 times that of the small sphere), the absorption amount is estimated like three curves in Figure S6. This is a graph of the large particle absorption amount based on two-component Asakura-Oosawa theory. The red thick curve shows the result when the large particle diameter is assumed to be 3 times that of the small particle and the black thin curve is assumed to be 2 times. The blue dashed line indicates the result when the large and the small particles are the same size, so there is no selectivity for absorption. Thus, the change in the absorption amount of decane (Figure 3) can be explained by a simple Asakura-Oosawa picture. Because we assumed spheres as guest molecules, we had to make a big difference between the large particle size and the small particle size to obtain the red thick line in Figure S6. However, as described in the main text, in the case of linear molecules, doubling the length approximately doubles the excluded volume, and the selectivity due to the difference in length will be enhanced, if compared to the spherical guest.

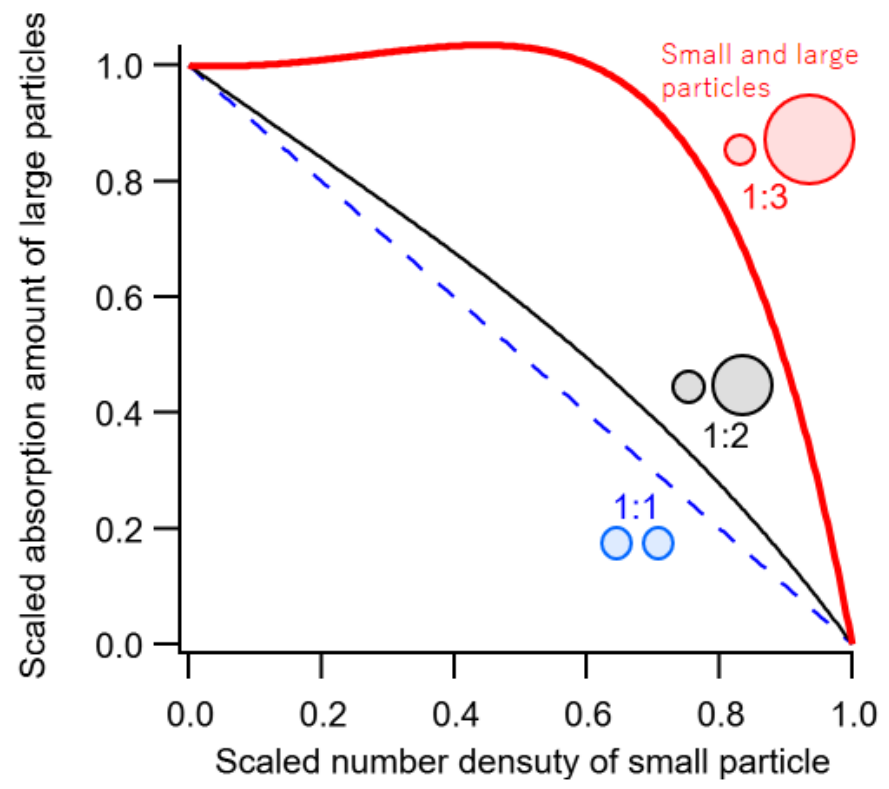

Figure S6. Estimate of large particle absorption amount at each diameter ratio of small particles and large particles by two-component Asakura-Oosawa theory. The ratio of the inner diameter of the tube to the diameter of the small particles was fixed at 5. Defining the diameter of small particles as $\sigma_{\mathrm{S}}$, the result shown by the red thick line can be obtained by the large particle diameter $3 \sigma_{\mathrm{S}}$ and the tube inner diameter $5 \sigma_{\mathrm{S}}$, and the total packing fraction 0.35 . The black thin line shows the result when the large particle diameter is assumed to be 2 times that of the small particle. The blue dashed line indicates the result when the large and small particles are the same size. 


\section{Supplementary equation S3 :}

\section{Linear dependence on $\rho_{s}$ of the effective potential between a guest and host}

In this section, we discuss the stability of a guest in a host. Here we adopt a model of a mixture of hard spheres of two different diameters as guest molecules. At the same time, a pore formed by the host molecules is approximated as a tube. Here we show that the stability of large particles in contact with the inner wall of the host tube increases linearly as the number of small particles increases under the condition that total packing fraction is constant.

At a same number density, the depletion interaction caused by the large depletants is larger than the depletion interaction caused by the small depletants. However, as the volume fraction of the small particles increases, the increase in the total number of particles results in the increase in the effective attraction between host and larger guest molecules. The reason is as follows. There is a relation $\phi_{\mathrm{L}}=\rho_{\mathrm{L}} \frac{\pi}{6} \sigma_{\mathrm{L}}^{3}$, where $\phi_{\mathrm{L}}$ and $\rho_{\mathrm{L}}$ are packing fraction and number density of the large particles, respectively. The subscript L should be replaced by $\mathrm{S}$ for small particles. When the total packing fraction $\phi_{\mathrm{t}}$ is constant, the effective potential between the host and the guest can be written as

$$
\begin{aligned}
& W_{\mathrm{L}}(\boldsymbol{x}) / k_{\mathrm{B}} \mathrm{T}=-\rho_{\mathrm{S}} \Delta V_{\mathrm{L}}^{\mathrm{S}}(\boldsymbol{x})-\rho_{\mathrm{L}} \Delta V_{\mathrm{L}}^{\mathrm{L}}(\boldsymbol{x}) \\
& \quad=-\phi_{\mathrm{t}}\left(\frac{6}{\pi \sigma_{\mathrm{L}}^{3}}\right)-\left[1-\left(\frac{\sigma_{\mathrm{S}}}{\sigma_{\mathrm{L}}}\right)^{3}\left(\frac{\Delta V_{\mathrm{L}}^{\mathrm{L}}(\boldsymbol{x})}{\Delta V_{\mathrm{L}}^{\mathrm{S}}(\boldsymbol{x})}\right)\right] \Delta V_{\mathrm{L}}^{\mathrm{S}}(\boldsymbol{x}) \cdot \rho_{\mathrm{S}},
\end{aligned}
$$

where $\Delta V_{L}^{S}$ is the excluded volume overlap when assuming small particles are depletant and a large particle is a guest, $\Delta \mathrm{V}_{\mathrm{L}}^{\mathrm{L}}$ is the excluded volume overlap when assuming large particles are depletant and a large particle is a guest, $\sigma_{\mathrm{S}}$ and $\sigma_{\mathrm{L}}$ are the diameters of small and large particles, respectively. Note that the superscripts indicate depletant, and the subscripts indicate guest. In the rightmost side of the above equation, the first term does not depend on $\rho_{\mathrm{s}}$ because $\phi_{\mathrm{t}}$ is constant, and the second term is proportional to $\rho_{\mathrm{s}}$ because both excluded volumes in the above equation do not depend on $\rho_{\mathrm{s}}$. Therefore, the condition for the guest to stabilize in the host as $\rho_{\mathrm{S}}$ increases is $\left(\frac{\sigma_{\mathrm{S}}}{\sigma_{\mathrm{L}}}\right)^{3}<\frac{\Delta \mathrm{V}_{\mathrm{L}}^{\mathrm{S}}}{\Delta \mathrm{V}_{\mathrm{L}}^{\mathrm{L}}}$. This condition is always met except when the guest size is zero. Thus, the effective potential between the guest and the host becomes linearly stronger as $\rho_{\mathrm{s}}$ is larger. 


\section{Supplementary equation S4: Simple theoretical calculation for Figure 3d}

In this section, in order to give a brief explanation for Figure 3d, we express the stability of large particles in contact with the inner wall as a function of the size of small particles under the condition that the volume fraction of small particles is fixed at $50 \%$. When the volume fraction of the small particles is fixed at $50 \%$, equation (2) in the main text can be written as

$$
W_{\mathrm{L}}(\boldsymbol{x}) / k_{\mathrm{B}} \mathrm{T}=-\frac{3}{\pi} \phi_{\mathrm{t}}\left(\frac{\Delta V_{\mathrm{L}}^{\mathrm{L}}(\boldsymbol{x})}{\sigma_{\mathrm{L}}^{3}}+\frac{\Delta V_{\mathrm{L}}^{\mathrm{S}}(\boldsymbol{x})}{\sigma_{\mathrm{S}}^{3}}\right) \text {. }
$$

Here, in addition to the condition $\phi_{\mathrm{t}}=$ const., let us fix the size of the large particles and consider the size dependence of the small particles. The first term on the right is constant. Thus the second term $\frac{\Delta V_{\mathrm{L}}^{\mathrm{S}}(\boldsymbol{x})}{\sigma_{\mathrm{S}}^{3}}$ determines the small particle size dependence of the effective attraction between large particles and the host. As the size of the small particles is reduced, the denominator decreases with the cube of the diameter. Although the numerator also has a dimension of volume, it is a "skin-like" volume at the host-guest interface. Therefore, as the diameter of the small particles is reduced, the amount of decrease of the numerator becomes smaller than the decrease of the denominator. As a conclusion, $\frac{\Delta V_{\mathrm{L}}^{\mathrm{S}}(\boldsymbol{x})}{\sigma_{\mathrm{S}}^{3}}$ becomes larger when $\sigma_{\mathrm{S}}$ becomes smaller. Thus, in a system where the volume fraction is constant (which is experimentally guaranteed in Figure 4), if the volume fraction is fixed at $50 \%$, the effective attraction between the large particle and the host becomes stronger as the smaller particles are smaller, resulting in the increase in the absorption of large particles. This will be an increase factor of long molecule absorption when the short molecules are shorter, shown in Figure $3 \mathrm{~d}$.

\section{Supplementary References}

(1) Uda, Y.; Kaneko, F; Kawaguchi, T. Selective Guest Uptake from Solvent Mixtures in the Clathrate Phase of Syndiotactic Polystyrene. Macromol. Rapid Commun. 2004, 25, 1900-1904.

(2) Silverstein, R. M.; Webster, F. X.; Kiemle, D. J. Spectrometric Identification of Organic Compounds, 2006, 7th Ed., Jhon Wily and Sons, Inc.

(3) Harada, I.; Takeuchi, H.; Sakakibara, M.; Matsuura, H.; Shimanouchi, T. Vibration Spectra and Rotational Isomerism of Chain Molecules. II. Butane, Pentane, Hexane, Pentane- $\mathrm{d}_{12}$, and Hexane- $\mathrm{d}_{14}$. Bull. Chem. Soc. Jpn, 1977, 50, 102-110.

(4) Akiyama, R.; Karino, Y.; Hagiwara, Y.; Kinoshita, M. Remarkable Solvent Effects on Depletion Interaction in Crowding Media: Analyses Using the Integral Equation Theories. J. Phys. Soc. Jpn. 2006, 75, 064804.

(5) Chandler, D. Introduction to Modern Statistical Mechanics, 1987, Oxford university press, Inc., New York. 